\title{
DRAFT: DESIGN OPTIMIZATION AND VALIDATION OF SINGLE-PHASE RECTANGULAR MICRO CHANNELS WITH AXIAL NON-UNIFORM WIDTH
}

\author{
Tijs Van Oevelen* \\ Applied Mechanics and Energy Conversion \\ Department of Mechanical Engineering \\ Katholieke Universiteit Leuven \\ Heverlee, Belgium \\ Email: tijs.vanoevelen@mech.kuleuven.be
}

\author{
Martine Baelmans \\ Applied Mechanics and Energy Conversion \\ Department of Mechanical Engineering \\ Katholieke Universiteit Leuven \\ Heverlee, Belgium \\ Email: martine.baelmans@mech.kuleuven.be
}

\begin{abstract} be achieved using non-uniform channels. non-uniform channels is lower than expected.

\section{NOMENCLATURE}

A Cross-sectional area

$C_{1}, C_{2}, C_{3}$ Constants used in Eq. (19)

c $\quad$ Specific heat capacity

$D \quad$ Hydraulic diameter

$f \quad$ Fanning friction factor

$H \quad$ Height

$h \quad$ Convection heat transfer coefficient

$J \quad$ Objective function

$k \quad$ Heat conductivity

$L \quad$ Length

$\dot{m} \quad$ Mass flow rate
\end{abstract}

A simple model for the simulation of the hydraulic behaviour and heat transfer of a single micro channel is formulated, assessed and optimized. This model is based on a thermally developing flow regime. The results of the optimization with this model show that a reduction of $7.6 \%$ in thermal resistance can

Furthermore, the assumptions of the simple model are validated by comparing the results with a more accurate, numerical model. It is shown that the simple model is sufficiently accurate to simulate the hydraulic and thermal behaviour of micro channels. The numerical simulations however show that the benefit of

*Address all correspondence to this author.

$\begin{array}{ll}\dot{m}_{\text {ref }} & \text { Mass flow rate scale } \\ P_{w} & \text { Wetted perimeter } \\ P_{h} & \text { Heated perimeter } \\ p & \text { Pressure } \\ \dot{Q}^{\prime \prime} & \text { Heat flux } \\ \operatorname{Re} & \text { Reynolds number } \\ T & \text { Temperature } \\ u & \text { Axial velocity component } \\ w & \text { Width } \\ x & \text { Axial coordinate }\end{array}$

\section{Greek symbols}

$\gamma \quad$ Constant used in Eq. (19)

$\theta$ Dimensionless temperature

$\mu \quad$ Dynamic viscosity

$v \quad$ Kinematic viscosity

$\rho \quad$ Mass density

$\tau_{w} \quad$ Wall shear stress

\begin{tabular}{ll}
\multicolumn{2}{l}{ Subscripts } \\
$c$ & Channel \\
$e$ & Element \\
fin & Fin \\
$f$ & Fluid \\
${ }_{\text {in }}$ & Inlet \\
out & Outlet \\
$w$ & Wall
\end{tabular}




\section{INTRODUCTION}

The vast increase in power densities of semiconductor electronics necessitate drastic changes in cooling solutions for future applications. At present, much research towards liquid and two-phase micro channel coolers for electronic components is ongoing. The idea of micro channel heat sinks was originally proposed by Tuckerman and Pease [1].

Performance characteristics of these coolers are largely dependent on geometry. Several authors [2-11] already tackled the problem of optimizing cooling performance of single-phase heat sinks by adjusting geometric parameters. Some applied numerical methods for modeling the flow and heat transfer; others worked with analytical models. Mostly, rectangular channels are optimized for minimal thermal resistance. The channel width, or equivalently the number of channels, is then used as design parameter. Both constant pressure drop and constant pumping power optimization are discussed in literature.

Only few applications of parameter shape optimization according to the definition by Bendsøe and Sigmund [12] have been performed. Bau [4] optimized the axial shape of a rectangular micro channel using an analytical model. This shape is represented by a quadratic polynomial. Fisher and Torrance [5] investigated the effects of channel boundary curvature to optimize the cross-sectional shape. They used a complex variable boundary element method to analyze the conjugate heat transfer.

We propose a design methodology for single-phase heat sinks. This design method comprises the optimization of singlephase micro channel heat sinks with axially non-uniform rectangular channels. This follows the idea by Bau [4], but is further improved and investigated. Heat sinks with non-uniform width channels benefit from a wide channel inlet region to allow sufficient mass flow, while keeping the maximal temperature at a low value by using a smaller outlet region.

The structure of this paper is as follows. The next section deals with the modeling aspects. Geometric definition of the micro channel as well as the physical modeling equations are provided there. In the third section, the optimization framework is introduced. This includes the objectives, constraints, parameterization and the solution method. Following, in the fourth section, the results of the optimization study are discussed for 4 specific cases. A numerical validation of these results is then presented in the fifth section. A comparison is made between the approximate, analytical model and the more accurate, numerical model. The results are also further assessed. In the concluding section, the major results and conclusions are summarized.

\section{MODELING}

In this section, a simple model that allows for the simulation of the fluid flow and heat transfer in a single, rectangular micro channel is derived. One of the most important model features is the ability to simulate the behaviour of a channel with non-
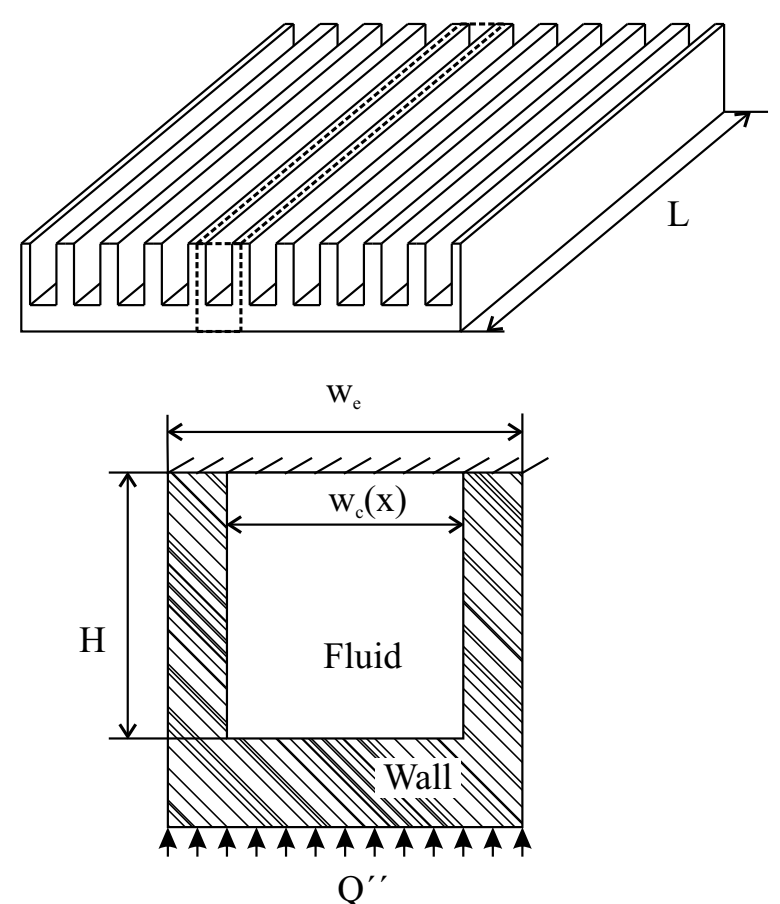

FIGURE 1. MICRO CHANNEL HEAT SINK AND SCHEMATIC DESCRIPTION OF THE CROSS-SECTION

uniform width. The model is a one-dimensional, integral model, meaning that the momentum and energy equations are integrated over the cross-section. The momentum equation is further integrated over the channel length.

\section{Geometry}

The research in this paper aims at micro channel heat sinks with rectangular channels. Such a heat sink is shown in Fig. 1. Heat is transferred from the chip through the bottom of the heat sink and convected away. The top of the heat sink is insulated.

Since micro channels are typically manufactured using milling or etching, the design is determined by the topview layout of the heat sink. The depth of the micro channels is fixed, but the width is adjustable. With these manufacturing processes, the designer has the freedom to let the channel width vary along the length of the channels.

The modeling in this paper is based on a single micro channel. The cross-section of such a channel is schematically shown in Fig. 1. Using a channel model implies that entrance and exit pressure losses due to the collectors of the heat sink are neglected. The analysis is thus based on the pressure drop over the micro channels only. Another implication is the assumption that the core of the heat sink behaves as if the heat sink is infinitely wide. Flow and heat transfer maldistribution near the sides of the heat sink are therefore neglected. 
Dimensionless parameters are distinguished from dimensional parameters by using a tilde, e.g. $\tilde{w}_{c}=w_{c} / H, \tilde{w}_{e}=w_{e} / H$, $\tilde{w}_{\text {fin }}=w_{\text {fin }} / H$ and $\tilde{x}=x / L . x$ is the axial coordinate of the channel.

Following equations can be used to determine the crosssectional area, the wetted and heated perimeter and the hydraulic diameter:

$$
\begin{aligned}
A & =w_{c} \cdot H=\tilde{w}_{c} \cdot H^{2} \\
P_{w} & =2 \cdot\left(H+w_{c}\right)=2 \cdot\left(1+\tilde{w}_{c}\right) \cdot H \\
P_{h} & =2 H+w_{c}=\left(2+\tilde{w}_{c}\right) \cdot H \\
D & =\frac{4 A}{P_{w}}=\frac{2 \tilde{w}_{c}}{1+\tilde{w}_{c}} \cdot H
\end{aligned}
$$

It should be noted that all these quantities depend on the axial coordinate, since the channel width is axially variable.

\section{Hydraulic model}

The hydraulic model is based on the integral axial momentum equation. An expression relating static pressure drop and mass flow rate is thereby established. The fluid flow in the micro channel is modelled as laminar and incompressible. This is a typical flow type in small-scale heat exchangers and heat sinks.

Following Rohsenow et al. [13] and Lee and Garimella [14, 15], the flow in the micro channel is assumed hydraulically developed, but thermally developing. Therefore, the hydraulically developed friction factor is used. The additional pressure drop due to contraction and expansion of the flow in the channel collector is not part of this model.

The total frictional pressure drop can be calculated by integration of the axial pressure gradient:

$$
\Delta p=-\int_{0}^{L} \frac{\partial p}{\partial x} \mathrm{~d} x
$$

This pressure gradient $\frac{\partial p}{\partial x}$ is related to the wall shear stress in the axial momentum balance.

$$
\frac{\partial p}{\partial x}=-\tau_{w} \cdot \frac{P_{w}}{A}
$$

Using the definitions of Fanning friction factor $\left(f=\frac{\tau_{w}}{\frac{\rho u_{m}^{2}}{2}}\right)$ and Reynolds number $\left(\operatorname{Re}=\frac{u_{m} D}{v}\right)$ and introducing the mass flow rate $\dot{m}=\rho u_{m} A$, where $u_{m}$ is the mean axial velocity, this equation can be written as

$$
\frac{\partial p}{\partial x}=-\frac{v}{8} \cdot \dot{m} \cdot f \operatorname{Re} \cdot \frac{P_{w}^{2}}{A^{3}}
$$

Substituting Eq. (7) in Eq. (5) and using the definitions from Eqs. (1) and (2), gives:

$$
\Delta p=\frac{v}{2} \cdot \dot{m} \cdot \int_{0}^{L} f \operatorname{Re} \frac{\left(H+w_{c}\right)^{2}}{w_{c}^{3} H^{3}} \mathrm{~d} x
$$

After rearranging this equation as an expression for $\dot{m}$ and introducing the dimensionless variables, an equation for the mass flow rate appears.

$$
\dot{m}=\frac{\Delta p H^{4}}{v L} \cdot\left(\frac{1}{2} \int_{0}^{1} f \operatorname{Re} \frac{\left(1+\tilde{w}_{c}\right)^{2}}{\tilde{w}_{c}^{3}} \mathrm{~d} \tilde{x}\right)^{-1}
$$

If we define the mass flow rate scale as $\dot{m}_{\text {ref }}=\frac{\Delta p H^{4}}{v L}$, then the equation for the dimensionless mass flow rate $\tilde{m}=\frac{\dot{m}}{\dot{m}_{\text {ref }}}$ is given by:

$$
\tilde{m}=\left(\frac{1}{2} \int_{0}^{1} f \operatorname{Re} \frac{\left(1+\tilde{w}_{c}\right)^{2}}{\tilde{w}_{c}^{3}} \mathrm{~d} \tilde{x}\right)^{-1}
$$

The same hydraulic model is retrieved by Bau in [4].

In order to model the $f$ Re product, the assumption made in [4] is used: a developed velocity profile is assumed at every axial location. In [4], it is argumented that this introduces only small errors if the change in velocity profile is gradual. This has previously been suggested by Harley et al. [16].

The correlation from Shah and London [17] for the dependence of $f \operatorname{Re}$ on the aspect ratio $\alpha$ is used:

$$
\begin{aligned}
f \operatorname{Re}= & 24\left(1-1.3553 \alpha+1.9467 \alpha^{2}-1.7012 \alpha^{3}\right. \\
& \left.+0.9564 \alpha^{4}-0.2537 \alpha^{5}\right), \quad \forall \alpha: 0 \leq \alpha \leq 1
\end{aligned}
$$

This correlation has a maximal error of $0.05 \%$.

\section{Thermal model}

The thermal model is used to calculate the temperature profile of the channel wall. Constant material properties are used and the heating due to viscous dissipation is neglected.

The first law of thermodynamics therefore gives an equation for the fluid bulk mean temperature:

$$
T_{f}(x)=T_{f, 0}+\frac{\dot{Q}^{\prime \prime} w_{e}}{\dot{m} c} x
$$


This is supplemented with the wall heat transfer equation,

$$
T_{w}(x)=T_{f}(x)+\frac{\dot{Q}^{\prime \prime} w_{e}}{h P_{h}}
$$

to obtain an equation for the wall temperature:

$$
T_{w}(x)=T_{f, 0}+\dot{Q}^{\prime \prime} w_{e} \cdot\left(\frac{1}{\dot{m} c} x+\frac{1}{h P_{h}}\right)
$$

Using the definition of Nusselt number $\left(\mathrm{Nu}=\frac{h D}{k}\right)$, Eqs. (3) and (4), we arrive at following expression:

$$
T_{w}(x)=T_{f, 0}+\dot{Q}^{\prime \prime} w_{e} \cdot\left(\frac{1}{\dot{m} c} x+\frac{2 \tilde{w}_{c}}{\operatorname{Nu} k\left(1+\tilde{w}_{c}\right)\left(2+\tilde{w}_{c}\right)}\right)
$$

which is made dimensionless using $\frac{\dot{Q}^{\prime \prime} H}{k}$ as a temperature scale, $\theta=\frac{T-T_{f, 0}}{\frac{\dot{Q}^{\prime \prime} H}{k}}$. With the definition of $\chi \equiv \frac{k L}{\dot{m}_{\mathrm{ref}} c}$, the group $\frac{k L}{\dot{m} c}$ can be written as $\frac{\chi}{m}$. The resulting model is given in Eq. (16) and is also used in [4].

$$
\theta_{w}(\tilde{x})=\tilde{w}_{e} \cdot\left(\frac{\chi}{\tilde{m}} \tilde{x}+\frac{2 \tilde{w}_{c}}{\operatorname{Nu}\left(1+\tilde{w}_{c}\right)\left(2+\tilde{w}_{c}\right)}\right)
$$

Note that the parameter $\chi$ can be written as:

$$
\chi=\mathrm{Be}^{-1}\left(\frac{H}{L}\right)^{-4}
$$

with $\mathrm{Be}=\frac{\Delta p L^{2}}{\mu \alpha}$, the Bejan number.

In other words, $\chi$ is related to the dimensionless plate spacing as defined by Bejan and Sciubba [2]:

$$
\delta \equiv\left(\frac{H}{L}\right) \mathrm{Be}^{1 / 4}=\chi^{-1 / 4}
$$

For the calculation of the local Nusselt number, it is assumed that the temperature profile is equal to that of a straight channel with the same development length $x$ and width $w_{c}$. The H1 thermal boundary condition is used, meaning axial uniform heat flux and circumferential constant temperature. This boundary condition was recommended by Lee et al. [14] as the most appropriate simplified boundary condition.
TABLE 1. CONSTANTS APPLIED TO EQ. (19) [19]

\begin{tabular}{llll}
$C_{1}$ & $C_{2}$ & $C_{3}$ & $\gamma$ \\
\hline 1 & 0.501 & 3.66 & $1 / 10$
\end{tabular}

An easy way for predicting the heat transfer in a channel with laminar thermally developing flow has long been a problem until recently. In the work by Muzychka, Yovanovich et al. $[18,19]$, a new framework is developed for correlating friction factors and Nusselt numbers, suitable for fully developed as well as developing flow types. These authors proposed the use of the square root of the cross-sectional area of the channel, $\sqrt{A}$, as the characteristic length scale for dimensionless properties, which allows for more easy and general correlations. They also succeeded in finding a correlation suitable for both the inlet region and the fully developed region, based on the Churchill-Usagi [20] asymptotic correlation method. In the current research, we only need their correlation for the thermally developing Nusselt number [19]. The subscript $\sqrt{A}$ denotes that the square root of the cross-sectional area is used as length scale.

$\mathrm{Nu}_{\sqrt{A}}\left(x_{\sqrt{A}}^{*}\right)=\left[\left\{C_{1} C_{2}\left(\frac{f \operatorname{Re}_{\sqrt{A}}}{x_{\sqrt{A}}^{*}}\right)^{\frac{1}{3}}\right\}^{5}+\left\{C_{3}\left(\frac{f \operatorname{Re}_{\sqrt{A}}}{8 \sqrt{\pi} \varepsilon^{\gamma}}\right)\right\}^{5}\right]^{\frac{1}{5}}$

Herein is $f \operatorname{Re}_{\sqrt{A}}$ the developed flow Poiseuille number with the square root of the cross-section as length scale. This is not calculated using the correlation by Shah and London, Eq. (11). To preserve consistency within the correlation, the corresponding equation by Muzychka and Yovanovich $[18,19]$ is used:

$$
\begin{aligned}
f \operatorname{Re}_{\sqrt{A}} & =8 \sqrt{\pi} g(\varepsilon) \\
g(\varepsilon) & =\left(\frac{\pi}{4} \frac{1+\varepsilon^{2}}{\sqrt{\varepsilon} \mathbf{E}\left(\sqrt{1-\varepsilon^{2}}\right)}\right) \\
& \approx\left[1.086957^{1-\varepsilon}\left(\sqrt{\varepsilon}-\varepsilon^{3 / 2}\right)+\varepsilon\right]^{-1}
\end{aligned}
$$

$x_{\sqrt{A}}^{*}=\frac{x}{\sqrt{A} \cdot P e}$ is the dimensionless coordinate used in the thermally developing heat transfer correlation. For rectangular channels, the variable $\varepsilon$ is equal to the aspect ratio $\alpha$.

The appropriate constants $C_{1}, C_{2}, C_{3}$ and $\gamma$ are given in Tab. 1 for the local Nusselt number of rectangular channels with uniform flux boundary condition. The accuracy of this correlation is in the range $[-7.03 \%, 9.93 \%]$ for high aspect ratio rectangular channels.

The correlation Eq. (19) returns the value for $\mathrm{Nu}_{\sqrt{A}}$. In the heat transfer model from this paper, the Nusselt number based on 
hydraulic diameter is used, so the correlation needs to be scaled by the factor $\frac{D}{\sqrt{A}}$.

Furthermore, as correlation (19) applies to a rectangular channel heated by 4 walls, it needs to be corrected for the present application with 3 heated walls. An easy way for this correction is a scaling with the ratio of the fully developed values. This method has been proposed by Phillips [21] and has also been applied and assessed by Lee and Garimella [15].

The resulting equation for determining $\mathrm{Nu}_{x, 3}$ is then:

$$
\mathrm{Nu}_{x, 3}\left(x^{*}\right)=\mathrm{Nu}_{\sqrt{A}}\left(x_{\sqrt{A}}^{*}\right) \cdot \frac{D}{\sqrt{A}} \cdot \frac{\mathrm{Nu}_{3}}{\mathrm{Nu}_{4}}
$$

where

$$
x_{\sqrt{A}}^{*}=x^{*} \cdot \frac{D}{\sqrt{A}}
$$

Correlations for the fully developed value of the Nusselt number for heating via 4 walls and 3 walls are found from Shah and London [17].

$$
\begin{aligned}
\mathrm{Nu}_{4}= & 8.235\left(1-2.0421 \alpha+3.0853 \alpha^{2}-2.4765 \alpha^{3}\right. \\
& \left.+1.0578 \alpha^{4}-0.1861 \alpha^{5}\right), \quad \forall \alpha: 0 \leq \alpha \leq 1 \\
\mathrm{Nu}_{3}= & 8.235\left(1-1.883 \alpha+3.767 \alpha^{2}-5.814 \alpha^{3}\right. \\
& \left.+5.361 \alpha^{4}-2 \alpha^{5}\right), \quad \forall \alpha: 0 \leq \alpha \leq 1
\end{aligned}
$$

Correlation (26) is also used as a reference in the thermally fully developed model.

\section{OPTIMIZATION}

This section deals with the optimization framework of the paper. Three items are covered in this section: problem specification, parameterization and solution method.

\section{Problem specification}

The optimization of the micro channel is performed with respect to minimal thermal resistance. This objective represents the desire to maximize the power density for a certain allowable temperature difference or vice versa. We assume that the overall temperature difference $\left(T_{j}^{\max }-T_{f, 0}\right)$ - with $T_{j}^{\max }$ the maximal junction temperature - is determined by the temperature difference between maximal wall and fluid inlet temperature
$\left(T_{w}^{\max }-T_{f, 0}\right)$. In dimensionless form, the corresponding objective function $J\left[\tilde{w}_{c}(\tilde{x}), \tilde{w}_{e}\right]$ can be written as follows:

$$
\begin{aligned}
& \min _{\tilde{w}_{c}(\tilde{x}), \tilde{w}_{e}} J\left[\tilde{w}_{c}(\tilde{x}), \tilde{w}_{e}\right] \\
= & \min _{\tilde{w}_{c}(\tilde{x}), \tilde{w}_{e}}\left(\max _{0 \leq \tilde{x} \leq 1} \theta_{w}(\tilde{x})\right)
\end{aligned}
$$

where Eq. (16) is used to calculate $\theta_{w}(\tilde{x})$.

It should be noted that not only the channel width distribution is optimized, but also the element width $\tilde{w}_{e}$. An explanation for this follows in the next section.

The objective function needs to be complemented with a constraint to make sure that the fins have a minimal width and can be formulated as:

$$
\begin{aligned}
\tilde{w}_{\text {fin }}(\tilde{x}) & =\tilde{w}_{e}-\tilde{w}_{c}(\tilde{x}) \\
& \geq \tilde{w}_{\text {fin }}^{\min }, \quad \forall \tilde{x} \in[0 ; 1]
\end{aligned}
$$

This constraint relates the element width $\tilde{w}_{e}$, that is representative for the surface area of the chip that is covered by one channel, to the local channel width $\tilde{w}_{c}(\tilde{x})$, which determines the flow and heat transfer characteristics of the micro channel.

Other constraints are already implicitly present in the modeling equations. For example, the allowable pressure drop can be regarded as a flow constraint. The constraint and variable are eliminated by substitution in the modeling equations. Similar reasoning applies to the chip heat flux and the inlet temperature.

\section{Parameterization}

The model derived in the preceding section, allows to simulate the flow and heat transfer in a rectangular micro channel with every axial width distribution one can imagine. It is however impractical to optimize a continuous width distribution, because this would mean that an infinite number of design variables need to be optimized. Therefore, the width profile of the channel is parameterized, using a spatial discretization with piece-wise linear interpolation between nodes:

$$
\begin{aligned}
\tilde{w}_{c}(\tilde{x}) & \leadsto \tilde{w}_{c} \in \mathbb{R}^{n} \\
\tilde{w}_{c} & =\left[\tilde{w}_{c}^{1}, \tilde{w}_{c}^{2}, \ldots \tilde{w}_{c}^{n} \mid \tilde{w}_{c}^{i}=\tilde{w}_{c}\left(\tilde{x}_{i}\right)\right]
\end{aligned}
$$

The parameter $\tilde{w}_{e}$ is a single number and does not need to be further parameterized. 


\section{Solution method}

A constrained optimization solver is used to solve the aforementioned optimization problem. However, the minimax form of the original problem (Eq. (27)) cannot be solved straight forward. Indeed, the objective function $J$ is not differentiable, and thus standard gradient-based optimization theory is not directly applicable. In order to facilitate the optimization, the thermal resistance optimization problem is reformulated. One extra variable $\hat{\theta}_{w}^{\max }$ and $n$ extra constraints (Eq. (32)) are added to the optimization problem description:

$$
\begin{aligned}
& \min _{\tilde{w}_{c}(\tilde{x}), \tilde{w}_{e}} J\left[\tilde{w}_{c}(\tilde{x})\right] \\
= & \min _{\tilde{w}_{c}(\tilde{x}), \tilde{w}_{e}, \hat{\theta}_{w}^{\max }} \hat{\theta}_{w}^{\max }
\end{aligned}
$$

subject to

$$
\begin{aligned}
\hat{\theta}_{w}^{\max } & \geq \theta_{w}(\tilde{x}), & & \forall \tilde{x} \in[0 ; 1] \\
\tilde{w}_{\text {fin }}(\tilde{x}) & \geq \tilde{w}_{\text {fin }}^{\min }, & & \forall \tilde{x} \in[0 ; 1]
\end{aligned}
$$

In the solution point of this new problem, the variable $\hat{\theta}_{w}^{\max }$ is equal to $\theta_{w}^{\max } \equiv \max \left(\theta_{w}(\tilde{x})\right)$, since $\hat{\theta}_{w}^{\max }$ is minimized without violating the constraint of Eq. (32). The new formulation of the optimization problem is therefore equivalent to the original formulation, but easier to solve.

The optimization problems are solved using the optimization routines from Matlab's Optimization Toolbox (The MathWorks Matlab R2009a). The routine fminimax automatically performs the reformulation necessary to solve the minimax problem.

\section{RESULTS AND DISCUSSION}

The method is tested for a practically realizable case. The appropriate parameters are listed in Tab. 2. The relevant dimensionless parameters that are used in the optimization are listed in Tab. 3. The reference mass flow rate $\dot{m}_{\text {ref }}$ is $0.0518 \mathrm{~kg} / \mathrm{s}$.

Four different set-ups are used for the optimization. The first set-up is the uniform channel optimization with the fully developed heat transfer model, Eq. (26) (UFD). In the second setup, the thermally developing heat transfer model, Eq (19) is used

TABLE 3. DIMENSIONLESS PARAMETERS

\begin{tabular}{ll}
$\tilde{w}_{\text {fin }}^{\min }$ & $\chi$ \\
\hline 0.1 & $3.316 \times 10^{-5}$ \\
\hline
\end{tabular}

(UTD). Optimization of a non-uniform channel is applied in the third (NUFD) and fourth set-up (NUTD), with respectively the fully developed and thermally developing heat transfer model.

Fig. 2 shows the optimized channel width distributions corresponding to the 4 optimization set-ups using a parameterization with $n=100$. Fig. 3 shows the corresponding wall temperature profiles.

A summary of the most important dimensionless data concerning these results is given in Tab. 4, including the element width $\tilde{w}_{e}$, the inlet $\tilde{w}_{c, \text { in }}$ and outlet width $\tilde{w}_{c \text {,out }}$, the maximal wall temperature $\theta_{w}^{\max }$, mass flow rate $\tilde{m}$, channel length $L^{*}$ and outlet Reynolds number $\mathrm{Re}_{\text {out }}$. The latter indicates that the flow is indeed laminar.

\section{Uniform width}

Looking at the optimal results for the uniform channels, we see that an optimal width has been found. Many authors have already argued that the optimal width of a uniform rectangular micro channels is the result of a balance between a capacitive thermal resistance and a convective thermal resistance. In the current model, these resistances are given by the two terms of Eq. (16):

$$
\begin{aligned}
\tilde{R}_{\text {cap }} & =\tilde{w}_{e} \cdot \frac{\chi}{\tilde{m}} \\
\tilde{R}_{\text {conv }} & =\tilde{w}_{e} \cdot \frac{2 \tilde{w}_{c}}{\mathrm{Nu}\left(1+\tilde{w}_{c}\right)\left(2+\tilde{w}_{c}\right)}
\end{aligned}
$$

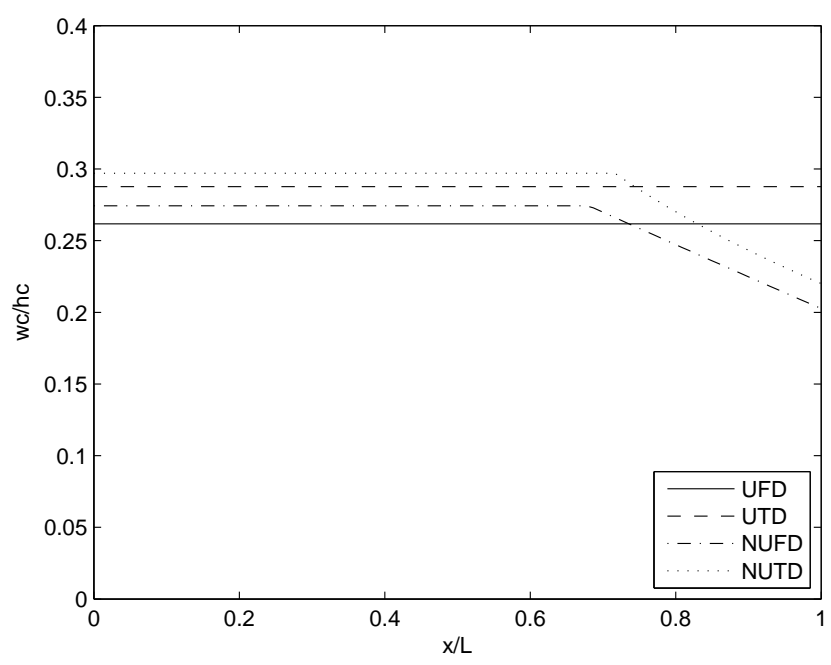

FIGURE 2. OPTIMAL CHANNEL SHAPES (U: UNIFORM WIDTH / NU: NON-UNIFORM WIDTH / FD: FULLY DEVELOPED MODEL / TD: THERMALLY DEVELOPING MODEL) 
TABLE 2. PARAMETERS OF THE TEST-CASE

\begin{tabular}{llllllll}
\hline$H(\mu m)$ & $w_{\mathrm{fin}}^{\min }(\mu m)$ & $L(m m)$ & $k\left(\frac{W}{m \cdot K}\right)$ & $c\left(\frac{J}{\mathrm{~kg} \cdot \mathrm{K}}\right)$ & $\rho\left(\frac{\mathrm{kg}}{\mathrm{m}^{3}}\right)$ & $\mu\left(\frac{\mathrm{N} \cdot \mathrm{s}}{\mathrm{m}^{2}}\right)$ & $\Delta p(\mathrm{kPa})$ \\
\hline 500 & 50 & 12 & 0.598 & 4180 & 998 & $1.004 \times 10^{-3}$ & 10 \\
\hline
\end{tabular}

\begin{tabular}{|c|c|c|c|c|}
\hline & UFD & UTD & NUFD & NUTD \\
\hline$\tilde{w}_{e}$ & 0.362 & 0.387 & 0.374 & 0.397 \\
\hline$\tilde{w}_{c, \text { in }}$ & 0.262 & 0.287 & 0.274 & 0.297 \\
\hline$\tilde{x}_{s}$ & - & - & 0.677 & 0.707 \\
\hline$\tilde{w}_{c, \text { out }}$ & 0.262 & 0.287 & 0.203 & 0.220 \\
\hline$\theta_{w}^{\max }$ & 0.0214 & 0.0211 & 0.0197 & 0.0195 \\
\hline$\tilde{m}$ & 0.00125 & 0.00162 & 0.00121 & 0.00152 \\
\hline$L^{*}$ & 0.0411 & 0.0295 & 0.0489 & 0.0368 \\
\hline $\operatorname{Re}_{\text {out }}$ & 204 & 260 & 207 & 257 \\
\hline
\end{tabular}

The capacative thermal resistance $\tilde{R}_{\text {cap }}$ is a decreasing function of $\tilde{w}_{c}$, due to the increase in mass flow rate when channel width increases. The convective thermal resistance $\tilde{R}_{\text {conv }}$ is an increasing function of $\tilde{w}_{c}$, because the heat transfer coefficient decreases when channel width increases. The optimal value of $\tilde{w}_{c}$ is therefore determined by the relative importance of both

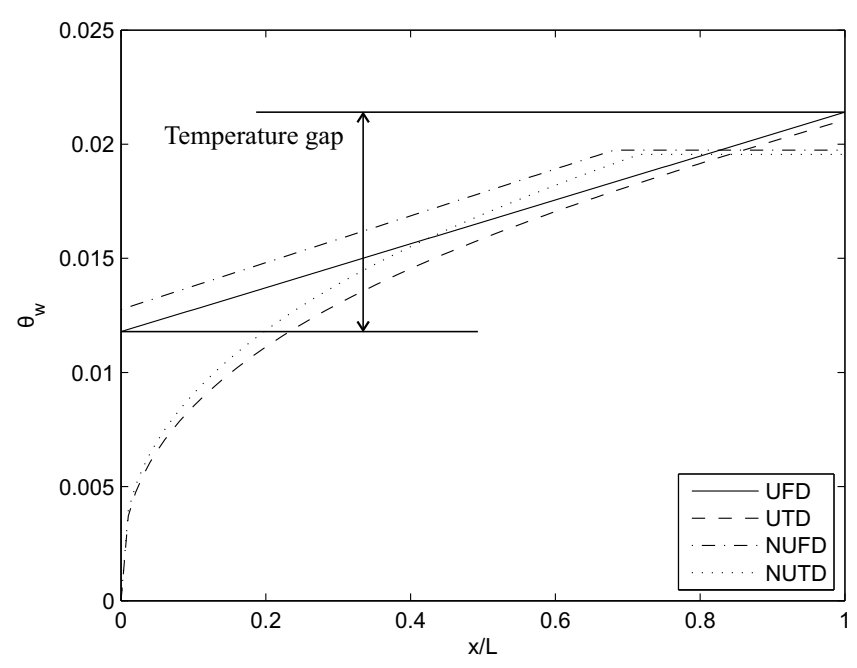

FIGURE 3. OPTIMAL TEMPERATURE PROFILES (U: UNIFORM WIDTH / NU: NON-UNIFORM WIDTH / FD: FULLY DEVELOPED MODEL / TD: THERMALLY DEVELOPING MODEL) thermal resistances.

Comparing the results of the fully developed (UFD) and the thermally developing model (UTD), we see that the optimal channel width is larger with the latter model. This is explained by the fact that the thermally developing Nusselt number is larger than the fully developed value. The order of magnitude of the values of $L^{*}$ in Tab. 4 shows that the entire channel is in the thermally developing region. The higher Nusselt number results in a lower convective thermal resistance for the UTD case. This shifts the balance towards a broader channel.

\section{Non-uniform width}

Going to the non-uniform channels, it should be noted that the element width $\tilde{w}_{e}$ plays a more important role here. In contrast to the uniform channels, the element width can no longer be substituted by $\tilde{w}_{c}+\tilde{w}_{\text {fin }}^{\min }$, because the channel width may vary along the length of the channel. This explains why $\tilde{w}_{e}$ is used as a variable in the optimization problem and why the constraint Eq. (33) is introduced.

From the results in Figs. 2 and 3, it may be seen that the constraints of Eqs. (32) and (33) determine the optimal result entirely. The fin width constraint Eq. (33) is active in the first part of the channel, limiting the channel width to a maximal value. In the second part of the channel is the maximal wall temperature constraint Eq. (32) active. This is expected because the fluid temperatures are highest near the outlet region.

The advantage of non-uniform channels compared to uniform channels is their ability to reduce the temperature gap between the local wall temperature $\theta_{w}(\tilde{x})$ and the maximal value that occurs in the channel $\theta_{w}^{\max }$. Figure 3 indicates the maximal temperature gap of the uniform fully developed channel. This temperature gap meausures the margin that is available to make the local channel width larger without affecting the thermal resistance. As mentioned before, this reduces the capacitive thermal resistance. Because the convective thermal resistance at the point of maximal wall temperature remains unaffected by this procedure, the total thermal resistance is also reduced. Logically, the optimal solution would occur when the wall temperature in the entire channel reaches the maximal value. Constraint Eq. (32) is then active in the entire channel. The corresponding optimal channel width distribution $\tilde{w}_{c}(\tilde{x})$ is a monotonically decreasing function of the axial coordinate $\tilde{x}$.

However, due to the constraint Eq. (33), this solution is not retrieved, as seen from Fig. 3. This constraint states that the channel width is restricted to $\tilde{w}_{e}-\tilde{w}_{\text {fin }}^{\min }$. Even without the specifica- 
tion of a minimal fin width does this constraint remain, because channel width cannot exceed the element width. The maximal value of the channel width is therefore determined by the element width.

The optimal element width $\tilde{w}_{e}$ on its turn is again determined by a balance between capacitive and convective thermal resistances. The capacitive thermal resistance decreases with $\tilde{w}_{e}$, because mass flow rate increases, while the convective thermal resistance increases due to the increase in heat load $\left(\dot{Q}^{\prime \prime} \tilde{w}_{e}\right)$ per channel.

Although the constraint Eq. (33) prevented from utilizing the full potential performance improvement, the temperature gap has been reduced, as can be seen in Fig. 3 .

This explains the resulting shape for non-uniform channels, which is entirely determined by the problem constraints. The intersection point $\tilde{x}_{s}$ is defined as the point where both constraints are active. This point is used to simply report the optimal channel shape in Tab. 4.

As before, larger channels are experienced for the thermally developing model.

Table 4 shows that the thermal resistance may be reduced by using non-uniform channels. The performance improvement predicted by the fully developed model is $7.9 \%$. According to the thermally developing model however, the improvement is only $7.6 \%$. The fully developed model does slightly overestimate the possible reduction in thermal resistance when going to non-uniform channels.

\section{NUMERICAL VALIDATION}

The results from the previous section are obtained with a simple analytical model. This model is spatially discretized to allow the optimization of the width distribution of the channel. The assumption that local velocity and temperature profiles are equal to those of a straight channel introduces an error and needs to be validated. Therefore, in this section the 4 resulting channels from the previous section are simulated with a CFD-model. The results of the analytical model are compared with that of the numerical model to judge its accuracy.

The set-up of the numerical model is discussed in the following part. Afterwards, the simulation results of the numerical and the analytical model are compared for both thermal and hydraulic characteristics. Therefore, the micro channels in the numerical simulation operate at the same mass flow rate as the analytical simulations. This way, the influence of modeling errors in the thermal and hydraulic model are seperated.

\section{Set-up}

The numerical model is set up in the commercial CFDpackage Fluent (Ansys Fluent 12.0) using the pressure-based solver. Both Navier-Stokes equations and energy equation are solved assuming laminar flow. The same constant properties as for the optimization are used for the coolant. Because of symmetry, only half of the channel is simulated.

The no-slip boundary condition is applied at the walls. The inlet condition for flow is the fully developed velocity profile approximation from Natarajan and Lakshmanan [22]:

$$
\frac{u}{u_{\max }}=\left[1-\left(\frac{y}{w_{c}}\right)^{n}\right]\left[1-\left(\frac{z}{H}\right)^{m}\right]
$$

The coefficients $m$ and $n$ depend on the aspect ratio of the channel as follows:

$$
\begin{aligned}
& m=1.7+0.5 \alpha^{-1.4} \\
& n= \begin{cases}2 & \text { if } \alpha \leq 1 / 3 \\
2+0.3(\alpha-1 / 3) & \text { if } \alpha \geq 1 / 3\end{cases}
\end{aligned}
$$

$u_{\max }$ can be found from the mean velocity $u_{m}$ after integration of Eq. (36):

$$
\frac{u_{\max }}{u_{m}}=\left(\frac{m+1}{m}\right)\left(\frac{n+1}{n}\right)
$$

The approximate $u_{\max } / u_{m}$ is within $0.9 \%$ of the exact value. $f$ Re numbers based on this profile are within $\pm 4.5 \%$ accuracy.

The other velocity components $v$ and $w$ are zero at the inlet. The channel outlet is modeled with an outflow boundary condition at uniform pressure. Since only half of the channel is simulated with the numerical model, a symmetry boundary condition is specified at $y=0$.

The inlet of the channel has a specified uniform temperature of $20^{\circ} \mathrm{C}$. A uniform wall heat flux is applied at the walls, except for the top wall which is adiabatic. The value of the wall heat flux depends on the specific case. Two remarks need to be made.

First, the desired thermal boundary condition type is the H1 condition, with axial uniform heat input and circumferential constant temperature. To obtain this, a small conductive layer is added to the walls with very high circumferential conductance and very low axial conductance. This method is also applied by Lee et al. [14]. Unfortunately, we did not yet succeed in a fully uniform circumferential temperature distribution. This will be improved in the final version of the paper by further increasing the circumferential heat conductivity.

Secondly, available boundary condition types in Fluent are not suitable for applying an axial uniform heat input. A uniform wall heat flux is the closest approximation, which is correct for uniform width channels, but slightly deviates in case of varyingwidth channels. The value of the wall heat flux $\dot{Q}_{w}^{\prime \prime}$ is chosen 
TABLE 5. BOUNDARY CONDITIONS

\begin{tabular}{lllll}
\hline & UFD & UTD & NUFD & NUTD \\
\hline$\dot{Q}_{w}^{\prime \prime}\left(\frac{W}{c m^{2}}\right)$ & 32.0 & 33.8 & 33.1 & 34.7 \\
\hline
\end{tabular}

TABLE 6. COMPARISON OF THERMAL RESISTANCE BETWEEN ANALYTICAL MODELS AND NUMERICAL MODEL

\begin{tabular}{lllll}
\hline & UFD & UTD & NUFD & NUTD \\
\hline$\theta_{w}^{\max }$ (numerical) & 0.0203 & 0.0201 & 0.0195 & 0.0193 \\
FD model (\%) & +5.17 & - & +1.37 & - \\
TD model (\%) & +4.34 & +4.60 & +2.29 & +1.43 \\
\hline
\end{tabular}

to match that of a chip with a heat flux $\dot{Q}^{\prime \prime}$ of $200 \frac{\mathrm{W}}{\mathrm{cm}^{2}}$. A small maldistribution of heat flow to the channel however remains giving an error on the local heat flux of maximum 3\%. The wall heat flux for each case can be found in Tab. 5 .

The same 4 channels as before were simulated. The shapes of non-uniform channels are simplified before modeling them. This is done by approximating the shape with two lines, specified by the numbers in Tab. 4. These small deviations on heat flow distribution and shape reconstruction will be improved for the final version.

The sizes of the grids that were used, are $200 \times 10 \times 20$ and $400 \times 10 \times 20$ respectively for the uniform channels and the nonuniform channels. A grid sensitivity study has been performed, proving that a grid independent solution is obtained.

\section{Evaluation of thermal modeling}

The accuracy of the thermal model is checked by comparing the predicted thermal resistance with the numerical simulation result. Table 6 gives the percentage difference in thermal resistance for the 4 different cases. The largest error on thermal resistance of the fully developed model is $5.17 \%$; the accuracy of the thermally developing model is even $4.6 \%$. We may conclude that both models are sufficiently accurate in predicting the thermal resistance.

For the final paper, another thermally developing Nusselt correlation will be tested that is reported to be more accurate. This is the correlation by Lee and Garimella [15], which is specifically derived for rectangular channels.

Furthermore, we may see from Tab. 6 that the effective thermal resistance is only slightly better when the thermally developing model is used in the optimization. The improvement for both a uniform channel as a non-uniform channel is about $1 \%$. This suggests that the simple model using fully developed heat transfer correlations may be used to optimize the shape of micro
TABLE 7. COMPARISON OF HYDRAULIC SIMULATION

\begin{tabular}{lllll}
\hline & UFD & UTD & NUFD & NUTD \\
\hline$\Delta p(k P a)$ & 9.89 & 9.93 & 10.47 & 10.52 \\
$\Delta p-\Delta p_{\text {target }}(k P a)$ & -0.11 & -0.07 & 0.47 & 0.52 \\
\hline
\end{tabular}

channel.

Nevertheless, the actual performance improvement when going to non-uniform width channels is reduced to only $4 \%$. This is only half of the predictions made by the analytical models.

\section{Evaluation of hydraulic modeling}

The difference between the numerical and the analytical model concerning the hydraulic modeling can be judged by looking at the pressure drop. The static pressure drop and absolute difference with the target of $10 \mathrm{kPa}$ is given for each case in Tab. 7.

Table 7 immediately shows that the pressure drop of the uniform channels is slightly lower than the target value. This difference is caused by the approximate inlet velocity profile, which shows to exhibit an underestimate of the wall shear stress. This explains the slightly lower pressure drop of the uniform channels.

The non-uniform channels however have a pressure drop which is higher than expected. The formerly explained phenomenon is thus overwhelmed by the additional pressure drop that is caused by redeveloping of the velocity profile when the channel width contracts. The pressure drop is apparently underpredicted in the analytical model by about $5 \%$. This is an important difference because correcting for this would decrease the mass flow rate and reduce the improvement that is found by going from a uniform channel to a non-uniform channel.

\section{CONCLUSIONS}

In this paper, a simple analytical model has been used to optimize the width distribution of a single-phase micro channel heat sink. The optimization is performed to find the width distribution with minimal thermal resistance for a specified pressure drop. Four different set-ups are optimized, for which the results have been extensively discussed. In addition, these results are compared with an accurate numerical model. Most important conclusions are summarized here:

- The analytical model using thermally developing correlations shows an improvement of $7.6 \%$ of a non-uniform channel compared to a uniform channel. The fully developed model slightly overestimates this value.

- Comparison with the numerical model shows that both the fully developed and the thermally developing model overestimate the actual thermal resistance with about 5\%. Further- 
more, the thermal resistance of the channels optimized with the thermally developing model were barely $1 \%$ lower than those optimized with the fully developed model. Both arguments suggest that the fully developed model may be used for shape optimization of a single-phase micro channel.

- Nevertheless, it is seen from the numerical simulations that the reduction of thermal resistance with non-uniform channels is only $4 \%$, while pressure drops have increased by 5 $6 \%$, due to the redeveloping of the flow. The benefit of nonuniform channels compared to uniform channels is apparently lower than expected.

\section{ACKNOWLEDGMENT}

This work is sponsored by the IWT, the agency for Innovation by Science and Technology, in Flanders, Belgium, through project SBO 60830, "Hypercool-IT" and PhD-grant SB 91326.

\section{REFERENCES}

[1] Tuckerman, D. B., and Pease, R. F. W., 1981. "Highperformance heat sinking for VLSI". IEEE Electron device letters, 2(5), May, pp. 126-129.

[2] Bejan, A., and Sciubba, E., 1992. "The optimal spacing of parallel plates cooled by forced convection". International Journal of Heat and Mass Transfer, 35(12), pp. 32593264.

[3] Yin, X., and Bau, H. H., 1997. "Micro heat exchangers consisting of pin arrays". ASME Journal of Electronic Packaging, 119, March, pp. 51-57.

[4] Bau, H. H., 1998. "Optimization of conduits' shape in micro heat exchangers". International Journal of Heat and Mass Transfer, 41, pp. 2717-2723.

[5] Fisher, T. S., and Torrance, K. E., 2000. "Constrained optimal duct shapes for conjugate laminar forced convection”. International Journal of Heat and Mass Transfer, 43, pp. 113-126.

[6] Ryu, J. H., Choi, D. H., and Kim, S. J., 2002. "Numerical optimization of the thermal performance of a microchannel heat sink". International Journal of Heat heat and Mass Transfer, 45, pp. 2823-2827.

[7] Stevens, T., Rogiers, F., and Baelmans, M., 2006. “Optimisation of micro-channel heat sink geometry". In 13th International heat transfer conference.

[8] Kim, D.-K., and Kim, S. J., 2007. "Closed-form correlations for thermal optimization of microchannels". International Journal of Heat and Mass Transfer, 50, pp. 53185322.

[9] Husain, A., and Kim, K.-Y., 2008. "Optimization of a microchannel heat sink with temperature dependent fluid properties". Applied thermal engineering, 28, pp. 11011107.
[10] Chen, C.-W., Lee, J.-J., and Kou, H.-S., 2008. "Optimum thermal design of microchannel heat sinks by the simulated annealing method". International communications in heat and mass transfer, 35, pp. 980-984.

[11] Van Oevelen, T., Rogiers, F., and Baelmans, M., 2009. "Optimal channel width distribution of single-phase microchannel heat sinks". In Proceedings of the 15th Therminic Workshop.

[12] Bendsøe, M. P., and Sigmund, O., 2004. Topology optimization: Theory, methods and applications, second ed. Springer Verlag, Berlin.

[13] Rohsenow, W. M., Hartnett, J. P., and Ganic, E. N., 1985. Handbook of Heat Transfer Applications, second ed. McGraw-Hill, New York.

[14] Lee, P.-S., Garimella, S. V., and Liu, D., 2005. "Investigation of heat transfer in rectangular microchannels". International Journal of Heat and Mass Transfer, 48, pp. 16881704.

[15] Lee, P.-S., and Garimella, S. V., 2006. “Thermally developing flow and heat transfer in rectangular microchannels of different aspect ratios". International Journal of Heat and Mass Transfer, 49, pp. 3060-3067.

[16] Harley, J. C., Huang, Y., Bau, H. H., and Zemel, J. N., 1995. "Gas flow in micro-channels". Journal of Fluid Mechanics, 284, pp. 257-274.

[17] Shah, R. K., and London, A. L., 1978. Laminar flow forced convection in ducts, a source book for compact heat exchanger analytical data. Advances in heat transfer, Supplement I. Academic Press, New York.

[18] Muzychka, Y. S., and Yovanovich, M. M., 1998. "Modeling friction factors in non-circular ducts for developing laminar flow". In 2nd AIAA Theoretical Fluid Mechanics Meeting, American Institue of Aeronautics and Astronautics.

[19] Muzychka, Y. S., and Yovanovich, M. M., 1998. "Modeling Nusselt numbers for thermally developing laminar flow in non-circular ducts". In 7th AIAA/ASME Joint Thermophysics and Heat Transfer Conference, American Institute of Aeronautics and Astronautics.

[20] Churchill, S. W., and Usagi, R., 1972. "A general expression for the correlation of rates of transfer and other phenomena”. American Institute of Chemical Engineers, 18 , pp. 1121-1128.

[21] Phillips, R. J., 1990. Microchannel heat sinks, Vol. 2 of Advances in thermal modeling of electronic components and systems. ASME, ch. 3, pp. 109-184.

[22] Natarajan, N. M., and Lakshmanan, S. M., 1972. "Laminar flow in rectangular ducts: Prediction of velocity profiles and friction factor". Indian Journal of Technology, 10(12), pp. 435-438. 